\title{
Flight Testing of Dynamic Soaring Part-1 : Leeward Inclined Circle Trajectory
}

\author{
Murat Bronz * 1, Nikola Gavrilović ${ }^{\dagger}{ }^{2}$, Antoine Drouin ${ }^{\star 1}$, Gautier Hattenberger ${ }^{\S 1}$, and Jean-Marc Moschetta ${ }^{\llbracket 2}$ \\ ${ }^{1}$ ENAC, University of Toulouse, F-31400 Toulouse, France \\ ${ }^{2}$ ISAE-SUPAERO, University of Toulouse, F-31400 Toulouse, France
}

\begin{abstract}
This paper presents flight experiments of dynamic soaring as an inclined circle trajectory on the leeward side of a ridge. Energy extraction through dynamic soaring can improve the range and endurance performance of fixed-wing vehicles on certain missions such as ocean monitoring, or flying over orographic wind sources. Real-life application of autonomous dynamic soaring presents challenges such as poor and limited sensor measurements, limited computation, estimation of the dynamic wind field. In this study, we assessed the feasibility of dynamic soaring in the shear layer with a small autonomous glider and showed the positive power contributing segments which are ascending into the positive wind gradient, and descending into the negative wind gradient. Flight test results revealed that, without any complex trajectories, on average, the glider can extract close to $60 \%$ of its required power from dynamic soaring. Additionally, a 6-DOF simulation environment is developed with a realistic aircraft model for the initial simulation of advanced trajectories. The results from the flight tests will serve as a database for the researchers to close the gap between simulations and the real flights to achieve a fully autonomous dynamic soaring.
\end{abstract}

Related data can be reached from : https://mrtbrnz.github.io/dynamic_soaring/

\section{Introduction}

Dynamic soaring is a flying technique that provides a positive contribution to aircraft power, leading to a significant enhancement in endurance and range for fixed-wing vehicles. The flight strategy can be applied to missions above the ocean surface or in the proximity of ridges, altogether, wherever a strong horizontal wind change with altitude is found. Seabirds such as albatrosses or petrels extract energy from the lower level of the atmospheric boundary layer over the ocean by climbing upwind into the increasing wind gradient and diving downwind into the decreasing gradient of the wind[1]. Researchers have been studying dynamic soaring [2,-4], especially with the use of remotely-piloted gliders [5, 6]. The most recent achievement for a high-speed dynamic soaring flight was demonstrated by Sachs et al., who simulated the feasibility of reaching close to $600 \mathrm{mph}$ flight speed without using a motor, with the help of dynamic soaring[7]. Zhao et al. investigated the optimal trajectories in atmospheric wind shears with altitude limitation, shears with changing direction, and also introduced a new idea of using negative shear layers occurring at high altitudes[8]. Deittert et al. simulated the effect of turbulence, modeled as Gaussian white noise, on the success of dynamic soaring of a small UAV with $3 \mathrm{~m}$ wingspan[9]. Bird et al. demonstrated a more complete dynamic soaring study by incorporating wind field estimation, trajectory planning, and path following solutions together with hardware in the loop system[10].

Besides the progress on understanding how dynamic soaring works, and how to maximize the energy extracted using a simulation environment, there has not been significant progress up to this date on a real-life application and flight testing. The main difficulties in real-life flights are low-quality sensor output, lack of accurate measurements of some quantities such as the angle of attack, or airspeed, and finally low computational power. We strongly believe that understanding the real-life challenges will lead researchers towards the development of more realistic simulation environments that are closer to real flights. Therefore, in this paper, we are presenting a dynamic soaring flight experiment of a leeward inclined circle trajectory. Additionally, the objective of this paper is to establish a first public

\footnotetext{
*Assistant Professor, ENAC F-31055 Toulouse, France, AIAA Member, e-mail: murat.bronz@enac.fr

$\dagger$ Post-Doc, Department of Aerodynamics, Energetics and Propulsion, 10 Avenue Edouard Belin, 31400 Toulouse, France, AIAA Member.

$¥$ Assistant Professor, ENAC F-31055 Toulouse, France

$\S$ Assistant Professor, ENAC F-31055 Toulouse, France

"Professor, Department of Aerodynamics, Energetic and Propulsion, 10 Avenue Edouard Belin, 31400 Toulouse, France, AIAA Member.
} 
database with experimental data, available for analysis and simulation applications. Special attention will be given to the imperfections in the measurements, variance, noise, and sampling rate of the flight logs.

\section{Related Research on Dynamic Soaring Flight Tests}

Besides having a vast amount of dynamic soaring related work done in a simulation environment, there have been very limited real-flight experiments presented. Mark Boslough [11, 12] demonstrated dynamic soaring flights by using custom built expanded polypropylene foam flying wings, called DS Beast. Custom data logging devices were designed and built by Jennings Engineering Inc. to record the flight data. The vehicles were equipped with GPS, 3-axis accelerometer, gyroscope, and magnetometer, and piloted manually by experienced RC-pilots to perform the dynamic soaring maneuver crossing the shear layer on the leeward side of a ridge. Several portions of the 22 minutes long flight data were presented, clearly showing the increase in the total energy during the dynamic soaring maneuver. The logs have shown the increase in airspeed, and therefore kinetic energy at each shear layer passage. The only other flight experiment including dynamic soaring maneuver has been presented by Gordon [13]. He performed 138 flights (88 test sorties and 50 training/avionics validation flights) with an L-23 full-size glider and gathered a sum of 27 hours of flight time. As a conclusion to his flight campaign, he has provided proof that dynamic soaring is achievable for full-size sailplanes.

As authors of this work, we are well aware of practical implication problems of using Dynamic Soaring for energy extraction during real-life missions, where the vehicle is required to fulfill a purposeful trajectory, such as flying from point A to B or surveying a given area. However, the challenges that have to be overcome to achieve autonomous dynamic soaring will certainly improve the overall capabilities of any fixed-wing vehicle (as well as a most rotary-wing vehicle).

Main contributions of this paper can be summarized as :

- An experimental demonstration of atmospheric energy-harvesting from horizontal wind gradient in form of repetitive circles.

- Sharing of a public database of dynamic soaring flight of a small glider.

- A 6-DOF simulation environment has been developed and also shared publicly with the objective of enabling faster progress by other researchers interested in the domain.

- As a measurement equipment, Smart-Probe (a multi-hole probe) has been developed alongside with this study.

All related data can be reached from : https://mrtbrnz.github.io/dynamic_soaring/

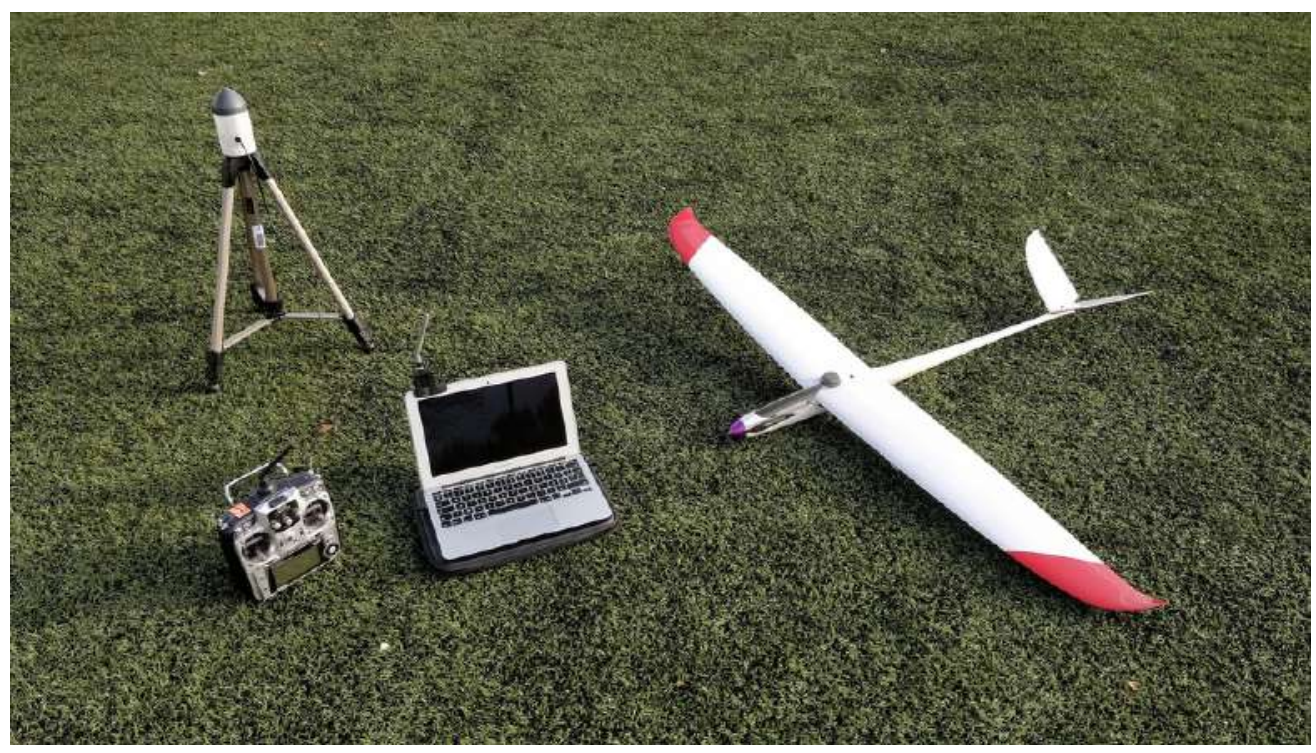

Fig. 1 System overview of the components used during the flight tests. 


\section{Test Equipment Overview}

In this section, we give an overview of the test equipment used during the flight experiments. The overall system, shown in Fig. 11, includes an electrically powered glider with $1.5 \mathrm{~m}$ wing span, equipped with Paparazzi Autopilot, a Ground Control Station (GCS) which is a regular laptop running Linux OS, an XBee radio-modem for data-link/telemetry communication, and a Futaba radio controller for safety-pilot to recover in the case of emergency. An optional Differential-GPS ground station is also shown in the Fig. 1 in case high accuracy position information is required.

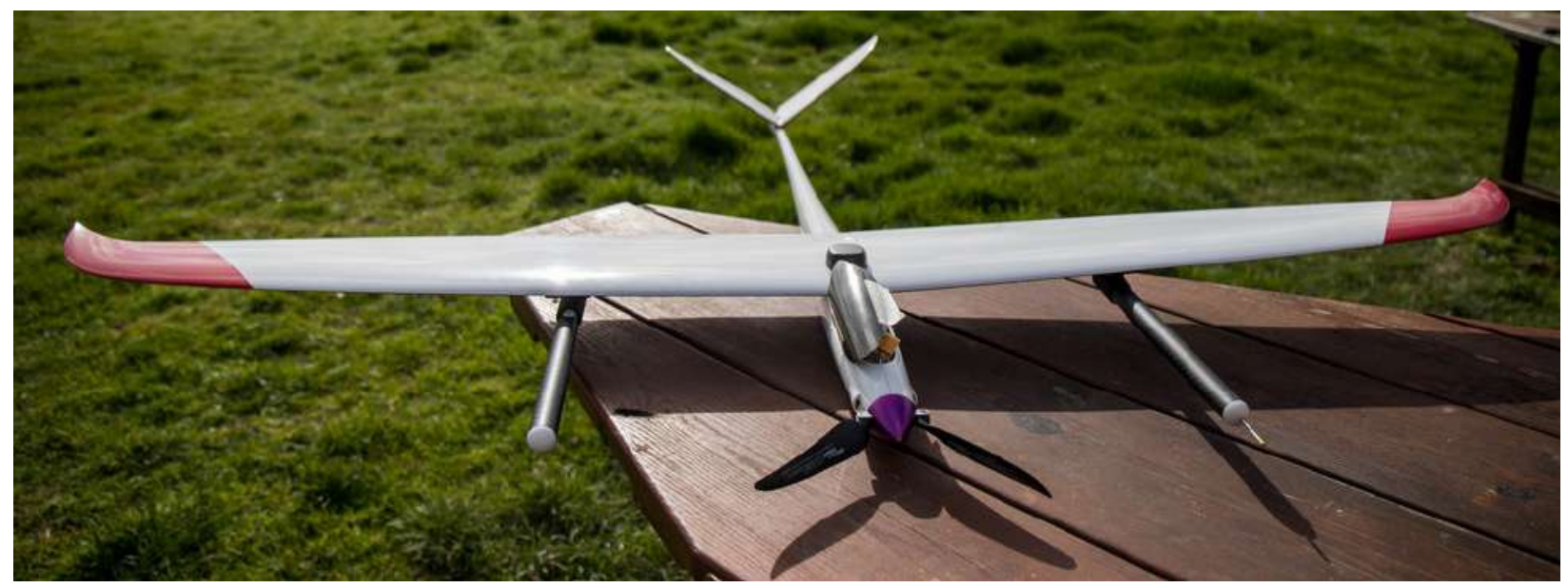

Fig. 2 A close-up view of the vehicle with Smart-Probe 5-hole probe is shown in the picture.

\section{A. Airframe}

Initial flight tests and previous work [14, 15] have been done by using EPP Foam Flying-wings, which are robust to harsh landings and easy to manipulate. However, the necessity of having a high-performance vehicle (i.e high lift to drag ratio) led us to select a composite glider. Table 1 shows the main specifications of the vehicle alongside with its components. Figure 2 shows a close-up view of the test vehicle. The additional carbon tubes that are attached

Table 1 Airframe Specifications

\begin{tabular}{lcl}
\hline \hline Specification & & Units \\
\hline Wing Span & 1.5 & {$[\mathrm{~m}]$} \\
Surface Area & 0.207 & {$\left[\mathrm{~m}^{2}\right]$} \\
Mass & 1.07 & {$[\mathrm{~kg}]$} \\
Battery Capacity & 19.0 & {$[\mathrm{Wh}]$} \\
Flight speed & 17 & {$[\mathrm{~m} / \mathrm{s}]$} \\
Flight time & 15 & {$[\mathrm{~min}]$} \\
\multicolumn{3}{c}{} \\
Components & & \\
Motor & \multicolumn{3}{l}{} \\
Autopilot & MVVS 2.0 - Kv 1600 \\
GPS & Uaparazzi Tawaki v1.0 & \\
\hline \hline
\end{tabular}

underneath the wing serve for angle of attack, side slip, and airspeed measurements. The one on the right wing is a 


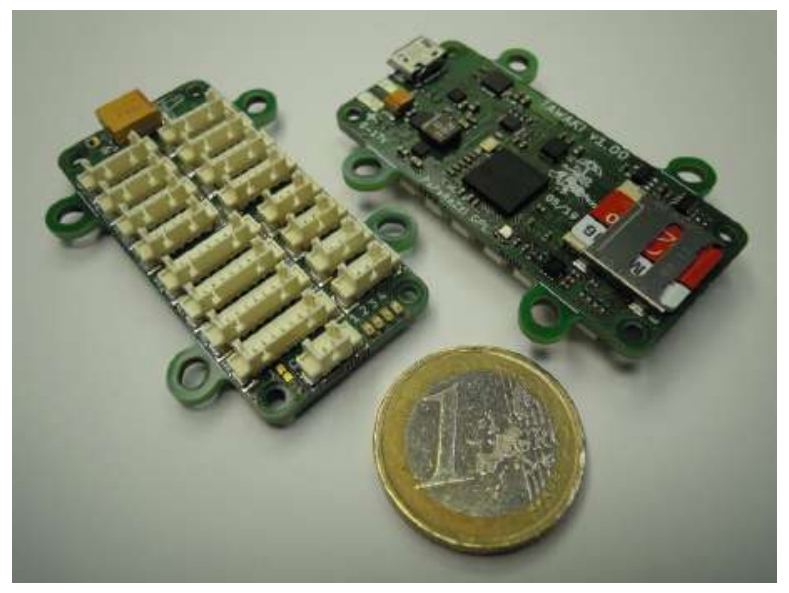

Fig. 3 3D view of the final design of the Tawaki autopilot

multi-hole probe, called Smart-Probe, and the one on the left is only an identical dummy tube in order to obtain the same amount of drag and mass to have equilibrium during the flight. The addition for mass and drag equilibrium was really necessary as the glider's vertical tail volume has been designed for nominal (i.e clean-wing) conditions.

\section{B. Autopilot System}

Throughout the whole flight tests, we have used the Paparazzi Autopilot system [16]. It is an open-sourced project started back in 2003 and used by several research groups, academics, and hobbyist. Being one of the first open-source autopilot systems in the world, Paparazzi covers all three segments: ground, airborne, and the communication link between them. Paparazzi has also its own complete flight plan language, where the user can define any possible trajectory using existing commands, such as circle, line, hippodrome, figure-eight, survey, etc. Additionally, any function written in $\mathrm{C}$ language can be called from the flight plan and executed. This opens up a lot of application possibilities, such as triggering a navigation procedure via a sensor output. Its integrated ground control station permits to control the flight plan execution, to move waypoints or change any parameters of the aircraft while in flight. No complex modifications were necessary to perform the dynamic soaring flight trajectories. Existing autonomous navigation routines have been used to follow the circular flight pattern, and additionally we have imposed an altitude reference that is varying with respect to the angular position of the glider on the circle. The resulting reference trajectory is an inclined-circles. The only human interaction from the ground control station was the high-level decision of inclination angle, altitude and position of the circle center, which were being interactively modified during the flight tests. In any phase of the flight, once the manual control is activated from the Safety RC-Transmitter, the safety pilot has the priority to take over the control of the aircraft and recover in case of emergency.

The test vehicle used the newest autopilot hardware of the Paparazzi project, named Tawaki. The Fig. 3 shows the top and bottom view of the Tawaki v1.0 autopilot. The general guidelines were to keep the board small, eventually with the same footprint as the previous generation for easier upgrade, improve the connectivity to support more sensors and use latest available integrated components such as MCU and IMU. The main characteristics of this new board, are listed in Table 2

\section{Smart-Probe}

One of the challenges during the flight experiments is to measure the angle of attack, side-slip angle, and airspeed accurately. That information will be necessary for both decision making and performance evaluation during dynamic soaring. Combined estimation of airspeed, angle off attack, and side-slip angle can be achieved using multiple wind vanes, measuring surface pressure difference on the wing, as shown in [14], or multi-hole pressure probes. Smart-Probe is a multi-hole probe that is developed in-house at ENAC. It has five holes circularly positioned on its semi-spherical tip. The main tube of Smart-Probe is made from carbon reinforced structure with the complete electronic hardware including pressure sensors integrated inside. The idea is to have a device that can gather and process data internally while directly delivering information to autopilot with a wired connection. Figure 4 a shows the Smart-Probe and the internal electronics can be seen in Fig. $4 \mathrm{~b}$. Table 3 presents the main specifications of integrated sensors within 
Table 2 General characteristics for the Tawaki v1.0 autopilot board

\begin{tabular}{lc}
\hline \hline Description & Details \\
\hline MCU & STM32F7 \\
IMU & ICM20600 (accel, gyro) + LIS3MDL (mag) \\
Baro & BMP3 \\
Serial & 3 UARTS, I2C (5V + 3.3V), SPI \\
Servo & 8 PWM/DShot output (+ ESC telemetry) \\
RC & 2 inputs: PPM, SBUS, Spektrum \\
AUX & 8 multi purpose auxiliary pins \\
& (ADC, timers, UART, flow control, GPIO, ...) \\
Logger & SD card slot \\
USB & DFU flash, mass storage, serial over USB \\
Power & 6V to 17V input (2-4S LiPo) \\
& 3.3V and 5V, 4A output \\
Weight & 12 grams \\
\hline \hline
\end{tabular}

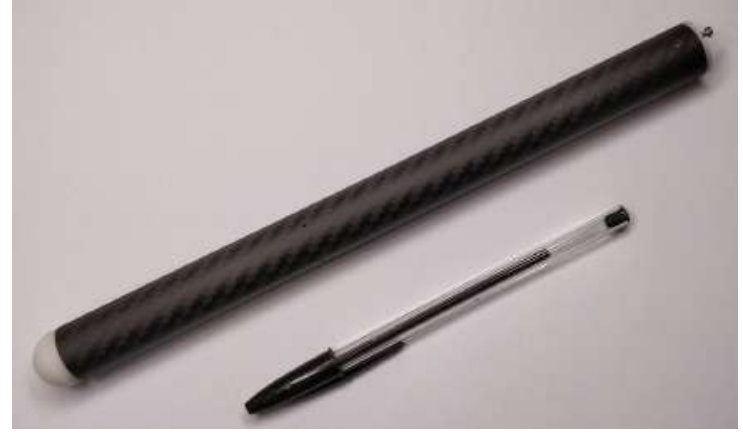

(a) Smart-Probe prototype assembled.

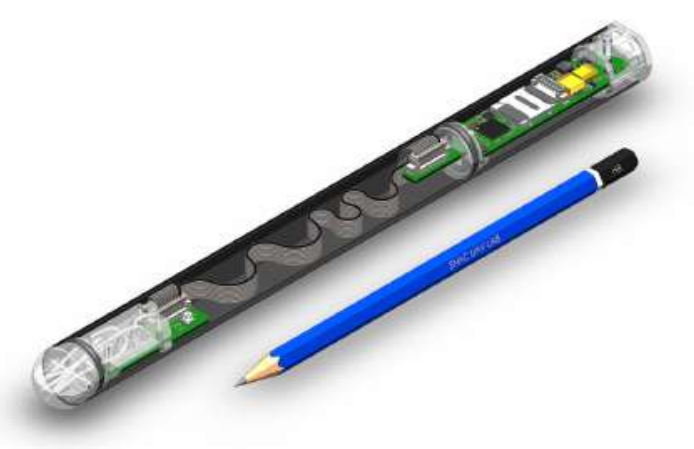

(b) Smart-Probe design with internal electronics.

Fig. 4 Smart-Probe multi-hole probe.

Smart-Probe.

\section{Smart-Probe Calibration}

The initial calibration process has been done inside a low speed wind-tunnel facility, where the test section dimensions were $120 \mathrm{~cm}$ by $80 \mathrm{~cm}$. The static ports on the probe are located at the very tip of the tube that has a semi-spherical shape. During the calibration process, it was important to keep the tip of the probe at the same location in the wind tunnel section (fluid stream tube) in order to avoid possible uncertainties that may arise from non-uniform spacial distribution of the flow. Therefore, a custom calibration bench has been used that holds the tip of the probe in the center of the wind-tunnel section during the pitch and yaw movements. Figure 5a shows the calibration mechanism inside wind tunnel's test section. The bench moves between $-20 \mathrm{deg}$, up to $+20 \mathrm{deg}$ both in the angle of attack and side slip axes. During calibration, first, the airspeed is fixed, and then for each angle of attack, the side-slip angle has been changed. Once all angle of attack and side-slip angles are covered, the wind tunnel speed has been changed and the process is repeated for different wind tunnel velocities $(7,11,15,20$, and $25 \mathrm{~m} / \mathrm{s})$.

A linear regression on the calibration data has been applied in order to estimate the angle of attack, side-slip angle 
Table 3 Main features and sensor specifications of the Smart-Probe.

\begin{tabular}{lc}
\hline \hline Description & Details \\
\hline MCU & STM32F7 \\
Differential pressure & $3 \times$ SDP31 \\
Absolute pressure & LPS33HW \\
IMU & ICM20600 (accel, gyro) \\
Logger & SD card slot \\
Data & UART, USB \\
Powering & $5 \mathrm{~V}$ \\
dimensions & $\varnothing 22 \mathrm{~mm}, \mathrm{~L} 110 \mathrm{~mm}$ min \\
\hline \hline
\end{tabular}

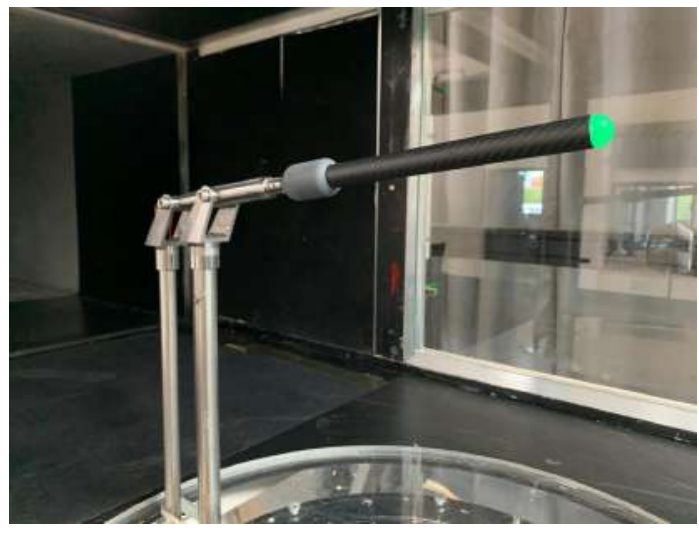

(a) Static calibration inside ISAE SABRE low-Re windtunnel.

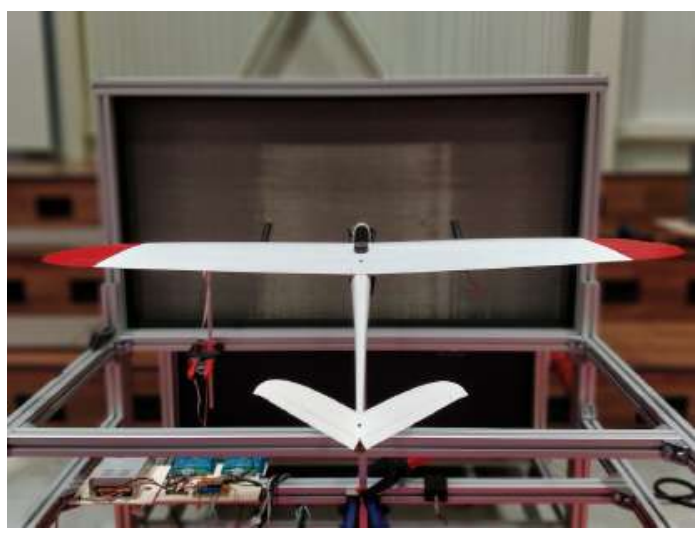

(b) Dynamic calibration and wing up-wash effect correction in front of ENAC WindShape.

Fig. 5 Two-stage calibration of Smart-Probe multi-hole probe measurement system.

and the dynamic pressure by using the three on-board differential pressure sensors as:

$$
\left[\begin{array}{l}
\alpha \\
\beta \\
q
\end{array}\right]=A\left[\begin{array}{l}
C_{1} \\
C_{2} \\
C_{3} \\
C_{1}^{2} \\
C_{2}^{2}
\end{array}\right]+\left[\begin{array}{l}
b_{1} \\
b_{2} \\
b_{3}
\end{array}\right], \quad \text { where, } \quad C_{1}=P_{1} / P_{3}, \quad C_{2}=P_{2} / P_{3}, \quad \text { and } \quad C_{3}=P_{3}
$$

where $A$ is a [3×5] coefficient matrix (obtained from the linear regression), and $b_{1-3}$ are the offset values, $P_{1}$ is the differential pressure between top and bottom holes, $P_{2}$ is the differential pressure between the right and left holes and the $P_{3}$ is the differential pressure between the center hole and the side holes that are situated $10 \mathrm{~cm}$ downstream with respect to the tip of the probe. As the angle of attack and side slip estimations will be a function of dynamic pressure, the linear regression features have been selected in a normalized way being $P_{1} / P_{3}$ and $P_{2} / P_{3}$. Figure 6 shows the result of the predicted angle of attack, side-slip angle and the dynamic pressure with respect to the wind tunnel reference that comes from the bench mechanism's encoders and wind-tunnel dynamic pressure sensors.

A second calibration was required once the probe is attached underneath the glider's wing. The circulation generated by the wing creates an up-wash which affects the measurements, mainly the angle of attack, of the probe. Figure $5 \mathrm{~b}$ shows an instant during the verification experiment that has been performed in front of WindShape, which is a wind generator that is used as a mobile wind-tunnel at ENAC. It is assumed that the open-jet stream of WindShape remains parallel with respect to the ground in front of the vehicle, therefore the on-board pitch measurements coming from the IMU can be used as the ground-truth for angle of attack. A linear correction factor of $c_{\alpha}=0.85$ was sufficient to obtain 

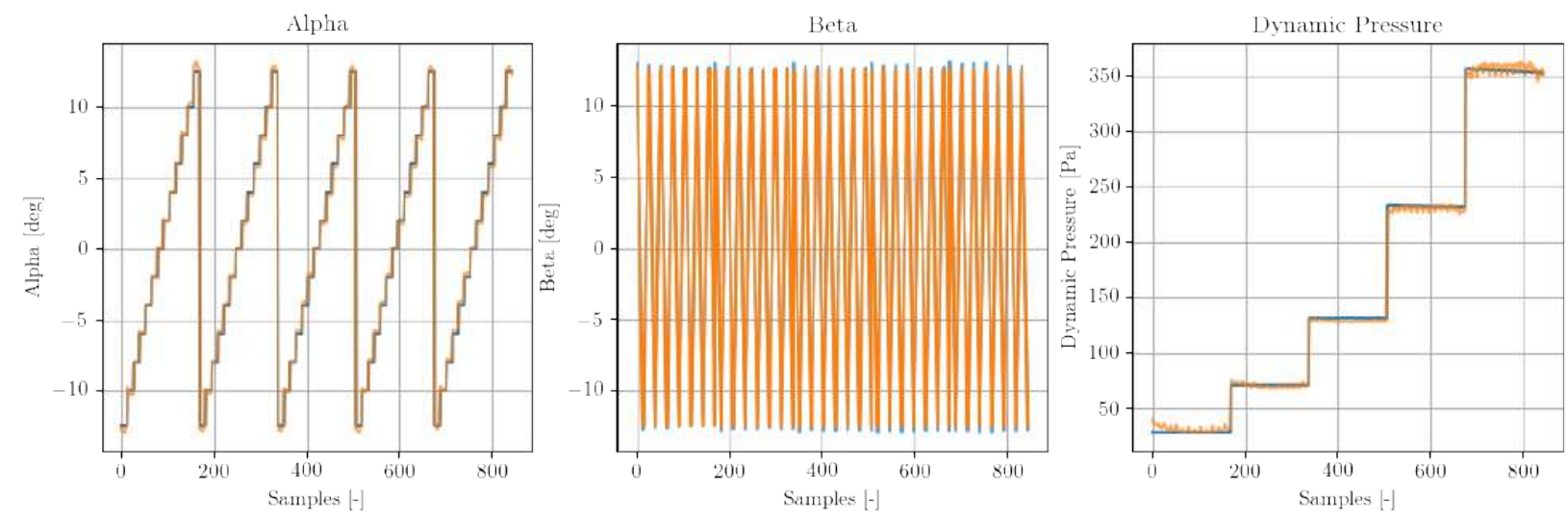

Fig. 6 Comparison of predicted versus ground truth values of angle of attack, side-slip angle and the dynamic pressure.

a good measurement with $\alpha_{\text {corrected }}=c_{\alpha} \alpha_{\text {measured }}$.

\section{Collected Data-set}

A dynamic soaring experimental campaign involves certain challenges that had to be overcome: flying in a proximity of a ridge, setting the infrastructure on the hardware, software, and human resources, and as well flight permissions. Therefore, as one of the main contribution of this work, we have open-sourced all of the flight test logs publicly which can be reached from : ( https://mrtbrnz.github.io/dynamic_soaring/). The different types of logs with their description are listed below:

GCS Log: Those quantities are logged inside the ground control station computer which is receiving messages from aircraft via telemetry. The use of small communication devices and portable antennas makes the system vulnerable to connection losses, therefore the GCS log can contain several missing information during the flight. Additionally, as the bandwidth of the telemetry is limited, the frequency of the messages is low. The main objective of the GCS log is to provide an insight into the major flight parameters, thus the data-set involved should mainly be considered for that purpose.

On-board SD Card Log: The limitation on the bandwidth and the low frequency is handled by logging the data on-board the autopilot, in a separate real-time operation system thread at a high frequency (i.e. $100 \mathrm{~Hz}$ ). The set of logging parameters can be defined before the flight with its corresponding frequency. The operator is required to download the binary log file after the flight and convert it to readable text file. Each message contains a time-stamp which is used to synchronize the whole data afterwards.

On-board Smart-Probe Log: During the flight tests, Smart-Probe records the sensory data at $500 \mathrm{~Hz}$. This is initially used to verify the quantities that are sent to autopilot. However, as high-frequency measurements may be useful for other reasons than autonomous dynamic soaring, this log will also be shared publicly alongside with other flight logs.

\section{Flight Tests}

Flight tests consist of performing inclined circles autonomously, while simultaneously measuring and calculating related flight parameters. All required changes in the reference path or its center location have been decided and modified by the human operator, through Paparazzi's ground control station, while the vehicle keeps following its current reference. Therefore, the main outcome of these presented flight tests and shared data will be on the quantities that have been experienced by the glider during inclined-circle flights. Figure 7 shows a sequential image of the glider climbing into the positive wind-gradient.

\section{A. Flight Test Procedure}

The flight test campaign begun in the late 2019, however only the latest flights include the electrical power measurements, therefore we will mostly concentrate on recent flights that provides complete information. The flight 


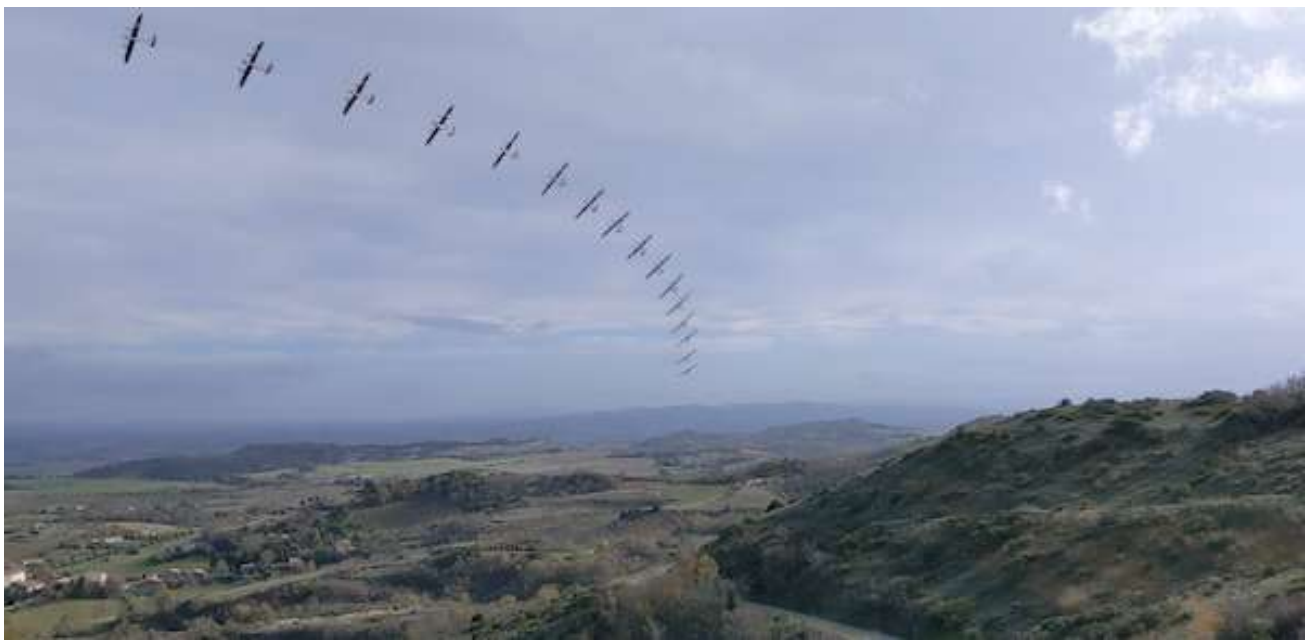

Fig. 7 Sequential imaging of the glider during the inclined circle trajectory, climbing upwind towards positive wind gradient.

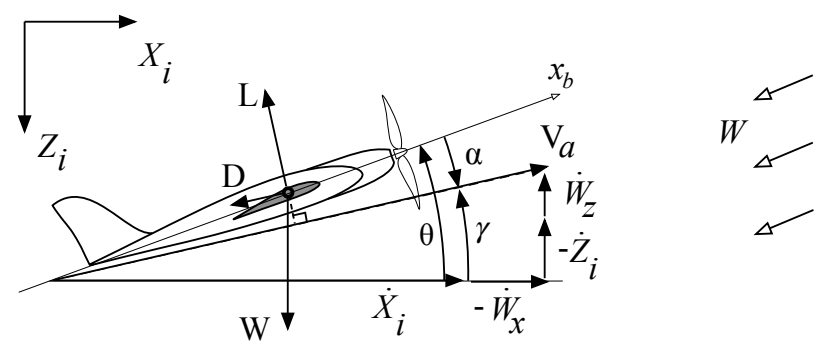

Fig. 8 Air-path relative velocity and longitudinal forces on a vehicle in uniform wind.

location chosen is in southern France, with coordinates of $43^{\circ} 27^{\prime} 44.8^{\prime \prime} \mathrm{N}-1^{\circ} 16^{\prime} 23.5^{\prime \prime} \mathrm{E}$. Typical flight procedure consists of taking-off from the top of the hill at $390 \mathrm{~m}$ ASL into the wind. Then initial climb and circle were being performed $50 \mathrm{~m}$ above ground level for one or two turns in order to go through safety-checks and verify GPS satellite acquisition. Afterwards, the inclined-circle flight block is activated with a circle center position placed at a known position.

Initial flight altitude is being kept at a safe height and then reduced progressively while watching the wind gradient variation during accent and descents through real-time telemetry data. Figure 10 shows the evolution of the altitude during a typical flight test.

\section{B. Measured and Calculated Quantities During Flight Tests}

The total air-path related energy of the vehicle can be given as the sum of inertial frame referenced potential energy plus, air-path referenced kinetic energy. It is important to note that it would be possible to undertake an analysis in which kinetic energy is defined with respect to groundspeed rather than to airspeed. However, the fundamental source of energy is the variation in wind speed that the vehicle encounters as it passes through the wind gradient. Moreover, it is the vehicle's airspeed and not the groundspeed which determines the aerodynamic force that is produced. Therefore, while it is possible to use both formulations to solve for flight trajectories that result in no net change in mechanical energy over a period of cyclical ascent and descent, the mechanism by which useful energy is gained must be understood in relation to the vehicle's movement relative to the air:

$$
E_{a}=m g z+\frac{1}{2} m V_{a}^{2},
$$

hence the specific energy rate of change with respect to time will be:

$$
\frac{\dot{E}_{a}}{m}=g \dot{z}+V_{a} \frac{d V_{a}}{d t} .
$$



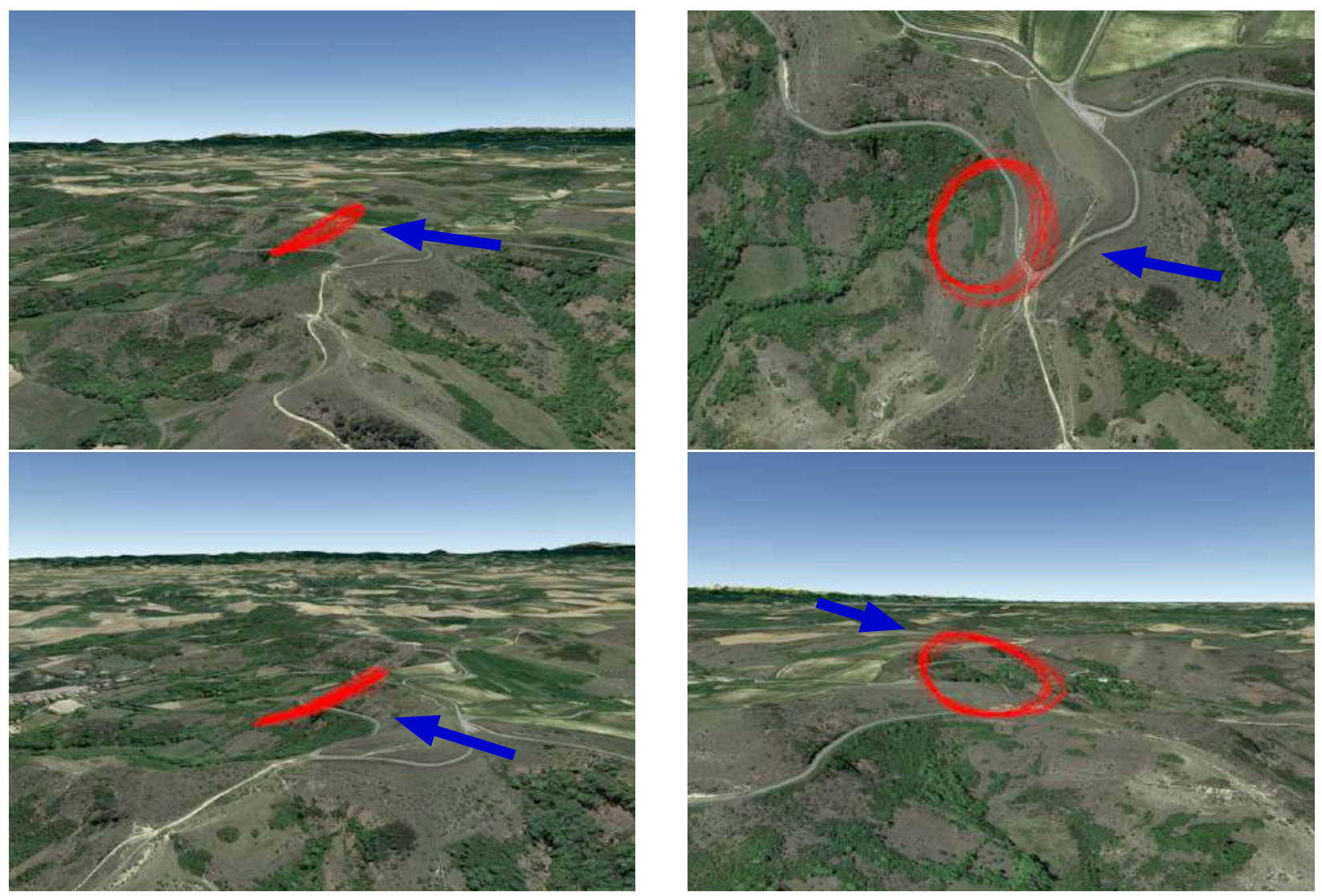

Fig. 9 Real flight trajectory of the inclined circle, controlled by Paparazzi Autopilot, direction of the wind is shown.

The equation of motion with wind components written in air-path reference

$$
T-D-W \sin \gamma=\frac{W}{g}\left(\dot{V}+\dot{w}_{x} \cos \gamma-\dot{w}_{z} \sin \gamma\right)
$$

If we represent the acceleration term including simplified expressions of lift, drag, and thrust with:

$$
L=q S C_{L}, \quad D=q S C_{D}, \quad \text { and } \quad T=q S C_{T},
$$

and with the new form of equation of motion which is:

$$
q \frac{S}{m}\left(C_{T}-C_{D}\right)-g \sin \gamma=\dot{V}_{a}+\dot{w}_{x} \cos \gamma-\dot{w}_{z} \sin \gamma,
$$

followed by the substitution described in [17], we have:

$$
\frac{d V}{d t}=-\frac{D}{m}-g \sin \gamma-\dot{w}_{x} \cos \gamma+\dot{w}_{z} \sin \gamma .
$$

Finally, the specific energy rate of change can be represented by:

$$
\frac{\dot{E}_{a}}{m}=-g w_{z}+\frac{q S}{m}\left(C_{T}-C_{D}\right) V_{a}-V_{a}\left(\dot{w}_{x} \cos \gamma-\dot{w}_{z} \sin \gamma\right) .
$$

The first term of the equation 8 states that energy can be gained with a negative component of vertical wind (static soaring within thermal or orographic updraft). It represents the rate at which gravitational potential energy is harvested when flying within an updraft $\left(w_{z}<0\right)$. This rate is independent on the flight, depending only on the strength of the 


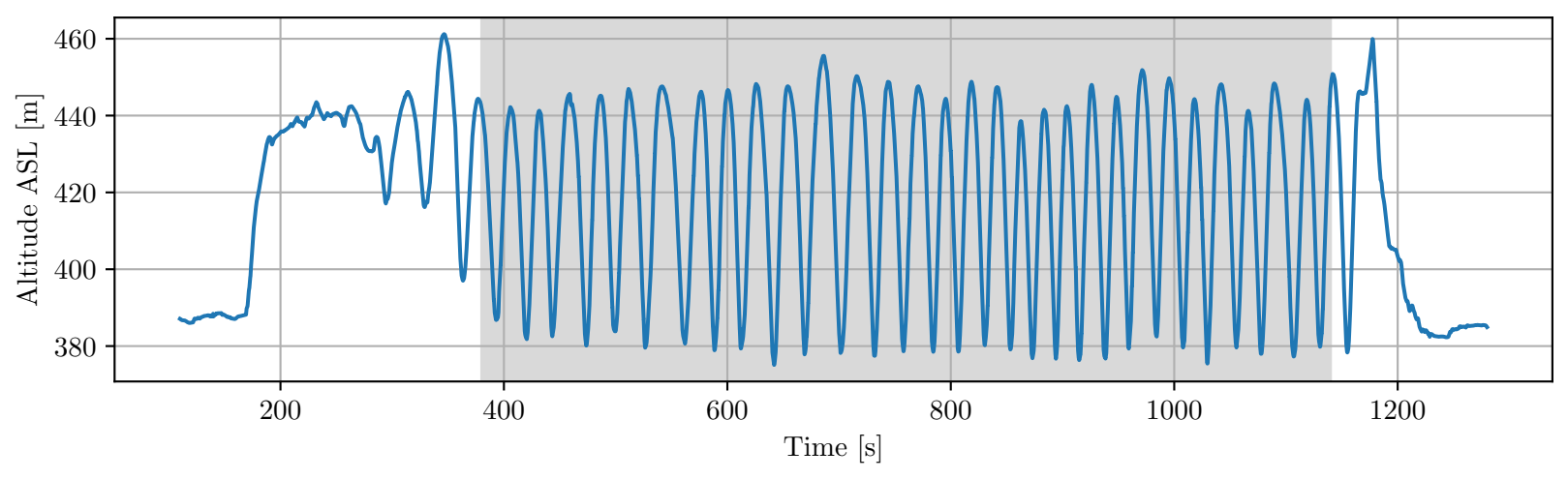

Fig. 10 Typical flight altitude during inclined circle flights.

updraft and weight of the vehicle. The most representative case of such an energy gain would be a soaring flight of birds in thermals, more particularly eagles. The second part illustrates power loss due to drag and invested power in form of thrust. Finally, the last term reveals the potential of wind gradients. With adequate control law, the goal of the dynamic soaring strategy would be to maximize and maintain the last term of equation 8 at a positive value, with respect to the optimal relation to the power required increase. Any action of the aircraft should preferably result in positive wind gradient power which out-weights the increase in power required. The third part of the equation 8 is isolated and taken for further integration. It reveals specific flight cases for which energy transfer between aircraft and the atmosphere is maximized. By analyzing wind derivatives in the longitudinal plane, we can distinguish some specific flight cases for maximization of energy gain. The wind field is assumed to be frozen in space, where $\nabla w$ is the spatial gradient of the wind vector, as shown in equation 9 .

$$
\frac{d w}{d t}=\nabla w\left[\begin{array}{c}
\dot{x}_{i} \\
\dot{z}_{i}
\end{array}\right]=\left[\begin{array}{ll}
\frac{\partial w_{x}}{\partial x} & \frac{\partial w_{x}}{\partial z} \\
\frac{\partial w_{z}}{\partial x} & \frac{\partial w_{z}}{\partial z}
\end{array}\right]\left[\begin{array}{c}
\dot{x}_{i} \\
\dot{z}_{i}
\end{array}\right]
$$

By substituting equation 9 into 8 , we introduce a new term which will be denoted as horizontal wind gradient power:

$$
P_{\dot{w}_{x}}=-V \dot{w}_{x} \cos \gamma=-V\left(\frac{\partial w_{x}}{\partial x} \dot{x}_{i}+\frac{\partial w_{x}}{\partial z} \dot{z}_{i}\right) \cos \gamma
$$

, vertical wind gradient power:

$$
P_{\dot{w}_{z}}=V \dot{w}_{z} \sin \gamma=V\left(\frac{\partial w_{z}}{\partial x} \dot{x}_{i}+\frac{\partial w_{z}}{\partial z} \dot{z}_{i}\right) \sin \gamma
$$

and finally, power required:

$$
P_{D}=-\frac{q S}{m} C_{D} V
$$

Analysis of different parts of previous equations and multiple derivations can bring the upcoming extreme cases, where specific flight maneuvers can be recognized, as shown by Gavrilovic et al. [18]. The equations reveal that climbing into positive wind shear and descending into a negative wind shear would increase the specific power of the aircraft, which will further be demonstrated in the experimental flight campaign.

\section{Measurement Imperfections}

It is important to mention that the real-flight measurements are very noisy and have to be filtered before any future use. Figure 11 shows the raw signal for airspeed and angle of attack compared to the filtered signal with a moving average filter with a sliding window size of 300 samples, resulting $3 s$ of time delay. In order to have synchronization between data, all measurements have been filtered with the same filter. 


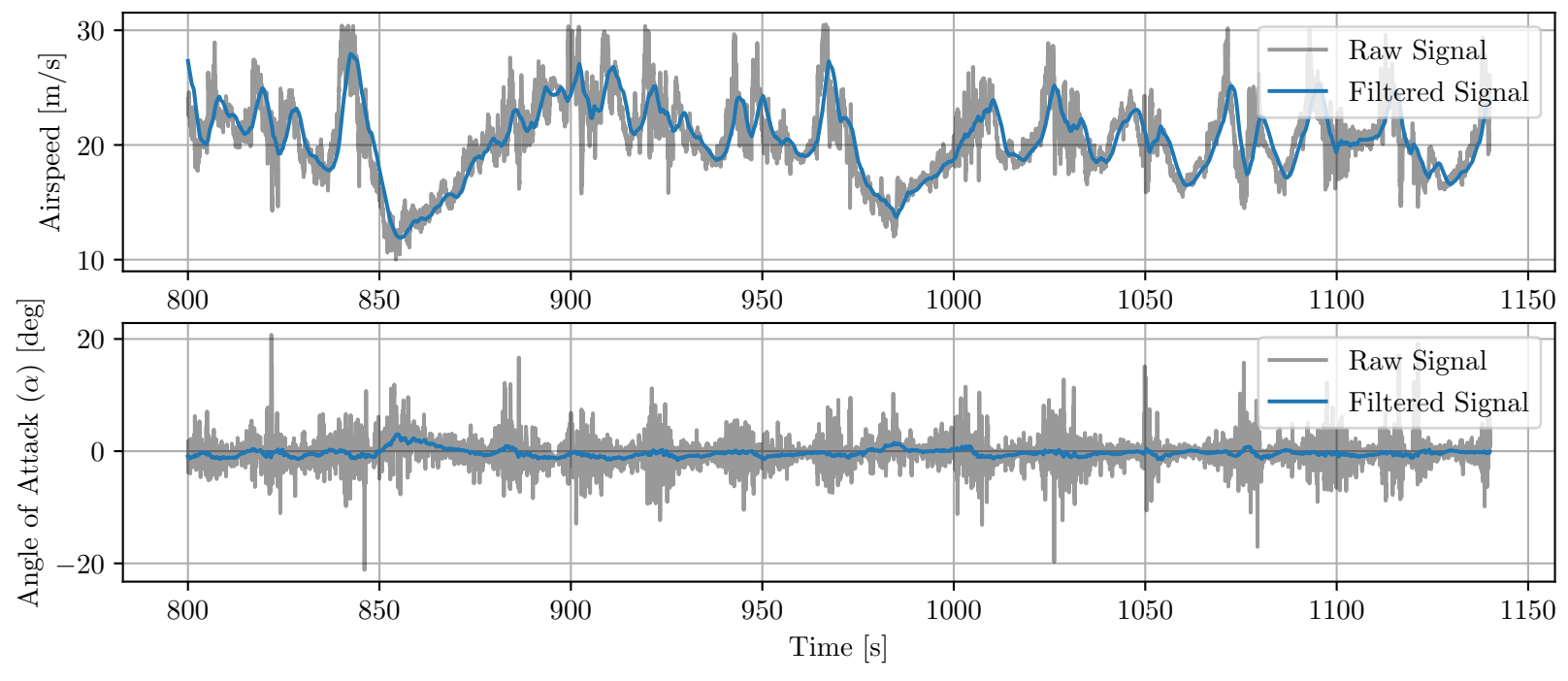

Fig. 11 Nature of measured airspeed and angle of attack signal from flights, compared to filtered signal.

\section{Results}

A full flight containing multiple inclined circles is shown in Fig. 9 where the powered glider flew on the leeward side of a hill. Although the flight from 20th of November 2020 consists of several full inclined circular trajectories, here we present only one of the full-cycles for a more detailed analysis and explanations. It can be seen on the graph that the glider climbs between initial altitude up to almost $40 \mathrm{~m}$ at the top of the inclined circle. The second plot from above shows the 3D-ground speed (which includes the vertical component combined with the horizontal components of the GPS measurements), and the on-board measured airspeed from two different sources. The first being the Smart-Probe and the second source is a traditional pitot-static tube mounted on the wing. The third plot from above shows the wind components and their derivatives estimated using the angle of attack measurements and airspeed from the Smart-Probe, pitch angle from IMU and ground and vertical speed from GPS. An augmentation of the axial in-plane wind component $w_{x}$ is apparent in the beginning of the climb phase, which is a sign of positive power contribution from horizontal wind gradient. However as it can be seen from the last plot, that the aircraft will be receiving an additional power from a throttle increased by the autopilot, trying to keep the reference altitude tracking error to minimum. Additionally, the extracted power from the wind fluctuation $P_{\dot{w}}$ is also plotted in two separate rows. Where $P_{\dot{w}}$ includes the contribution of both $\dot{w}_{x}$ and $\dot{w}_{z}$ as in equation 10

The aircraft has been also recently equipped with an electrical current sensor, in order to obtain the electrical power consumption. This information is used to estimate the aerodynamic power input coming from propeller thrust by using an empirical relation based on throttle percentage and electrical power measurements. The glider performs the full circle with an airspeed that varies between $21 \mathrm{~m} / \mathrm{s}$ and $27 \mathrm{~m} / \mathrm{s}$ as it can be seen in the second graph of Fig. 12 from top. It is possible to assume that total propulsion efficiency of the system remains constant in this range, and also below a certain throttle percentage, the propeller does not generate positive thrust. Then isolating only the positive contributing electrical power portion, and applying constant propulsion efficiency of $\eta_{p}=0.4$, will result the shaded area that is shown at the bottom of Fig. 12. The integration of this grey area results the estimated positive aerodynamic power contribution $\left(\sim \sum P_{\text {Aero }}\right)$ coming from the propulsion system.

It can be seen that the sum of estimated drag generated power $\sim \sum P_{D}$ is very close to the sum of estimated aerodynamic power $\sum P_{\text {Aero }}$ and the sum of wind gradient based dynamic soaring power $\sum P_{\dot{w}_{x}}$ over the full circle. This can be also seen on a longer period of flight such as shown in Fig. 15] with two consecutive circles and Fig. 16 with fourteen consecutive inclined circles. The final objective of the ongoing experimental campaign will be to achieve neutral and positive energy cycles where the invested power will not be needed. 

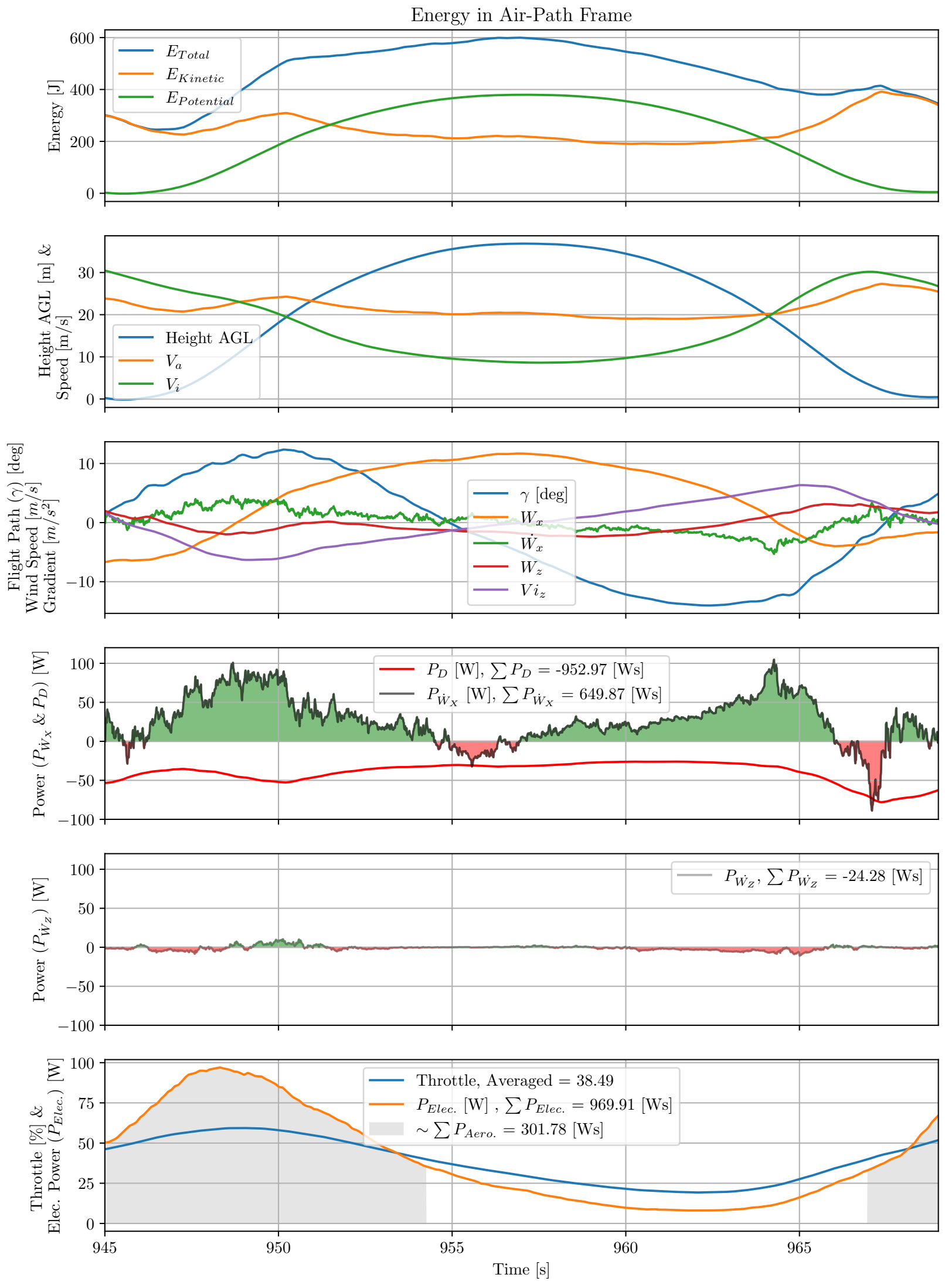

Fig. 12 Energy cycle and wind gradient power during a full cycle with emphasis on the contribution of horizontal wind gradient $\dot{w}_{x}$ during ascend towards positive wind gradient and descend towards negative wind gradient. 


\section{Simulation Environment}

Following the presented experimental flights, it is beneficial to develop more advanced control algorithms in a simulation environment. However, the main decision while developing a simulation framework is the compromise between the level of complexity and the representation of real-physics of the problem. The main objective is to keep it as simple as possible while retaining realism. Here, as the level of "realness" is already known from the flight experiments, the simulation environment is being tweaked up to the point where it can capture every important detail that can be experienced during flight tests.

Therefore, a new simulation environment, called Python Aerospace Toolbox (PAT), has been developed and made publicly available (https://github.com/poine/pat) under an open-source license in the hope that it will enable the community to verify and further improve the presented results.

The simulation environment comprises:

- A high accuracy 6-DOF dynamics model:

Several common mathematical representations are available for the state vector of the solid, such as Euler angles or quaternion for the orientation, and aero, body, or world-referenced velocities, providing a trade-off between numerical accuracy and ease of implementation. Actuators are modeled as saturated linear first-order systems.

- Automatic generation of aerodynamic coefficients via AVL from input aircraft geometry:

A standard linear aerodynamic model is implemented [19]. The aircraft steady aerodynamic coefficients have been determined using the Athena Vortex Lattice (AVL) program designed by Professor Mark Drela from Massachusetts Institute of Technology. The software has been modified to take into account the viscous effects. Based on Bronz [20], each section of the wing is treated according to its Reynolds number and corresponding viscous polar. The viscous drag coefficient in this study has been taken as a function of the total angle of attack seen by each section and the corresponding Reynolds number.

- Atmospheric models included for different scenarios:

We provide support for common analytical models such as thermals, flow around ridge, and also numerical grid models in the NETCDF4 format. Such numerical grids can be produced by meteorological simulations.
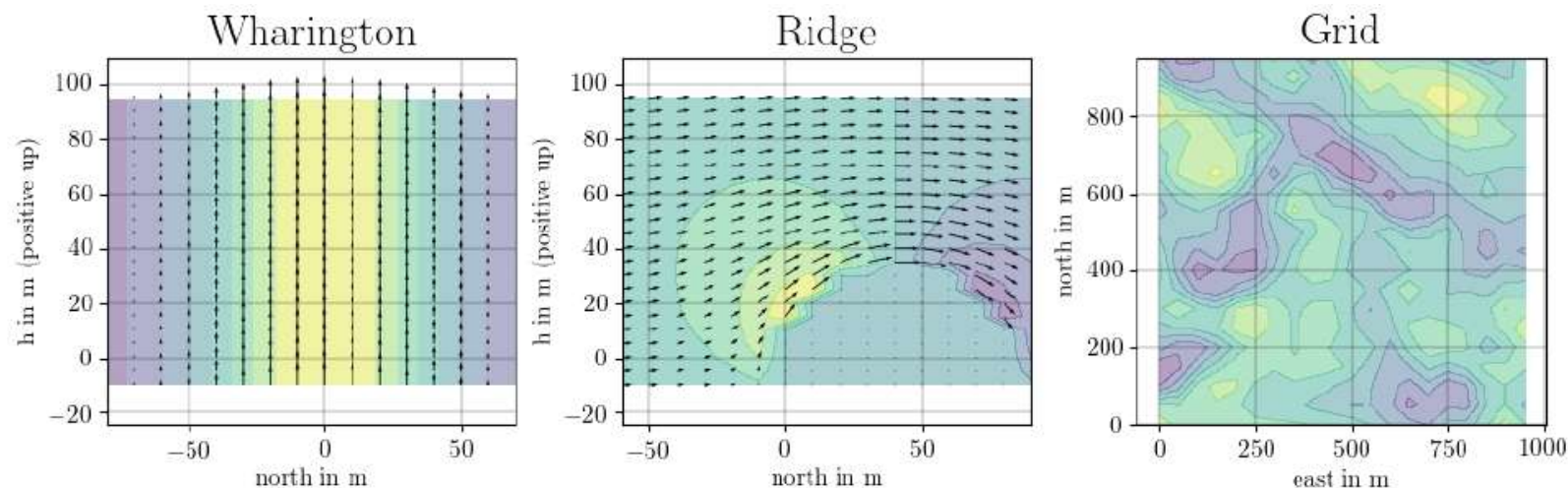

Fig. 13 Atmosphere models: Left, Wharington thermal model[21], center Ridge model[22], right Grid Model[23].

\section{A. Current Capabilities}

In its current state, the provided simulator environment can perform basic trajectories such as line, circle, oval, figure-eight, including inclined circle trajectory as used in the presented flight tests. All of the mentioned trajectories can be performed inside a virtual atmosphere defined analytically, or using data-driven methods. Figure 13 shows some of the existing and supported atmosphere model examples.

Successful dynamic soaring maneuver requires a significant difference on the wind speed in the shear layer. Basic flight control laws applied in most of the fixed-wing vehicles treats the wind as a perturbation. In order to follow the desired trajectory in such varying wind field, the control laws can no longer assume the wind as a perturbation. However,

†http://web.mit.edu/drela/Public/web/avl/ 

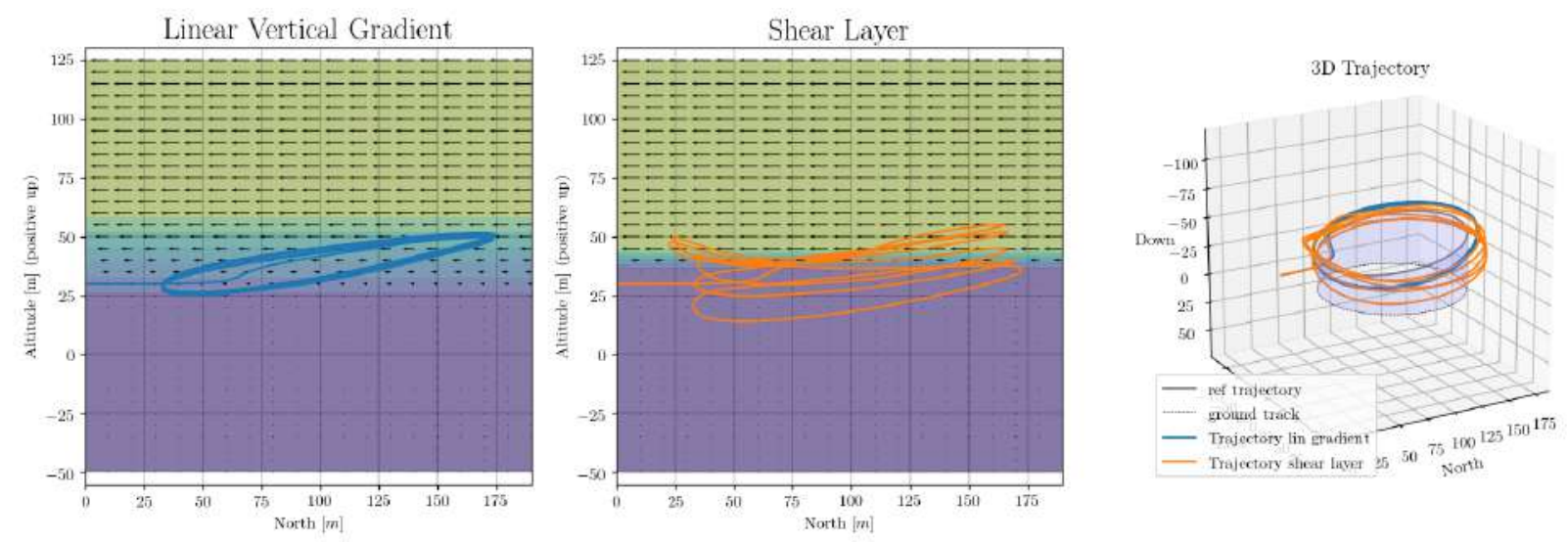

Fig. 14 Simulation of inclined circle trajectory inside linear vertical wind gradient on the left, thin wind shear layer in the middle, and the 3D trajectories of the two cases with respect to desired reference trajectory is shown.

a first set of classic guidance and piloting command laws have been developed as to mimic those available in Paparazzi Autopilot System.

- The piloting law is comprised of quasi linear reference model [24] driving linear feedback and feed-forward laws.

- The horizontal guidance law is a classic pure pursuit and the vertical guidance law is a cascade of PIDs.

Existing control loops were rapidly tuned by hand, with the exception of the piloting loop which was given an algebraic solution and numerically expressed using system identification, as to maintain the realism of the simulation. It should be noted that existing control structure only handles wind as a perturbation, as opposed to planning for it. Guidance methods which take into account the wind state are generally computationally demanding. As a future work, the use of vector field guidance [25], which offers superior properties and less computational power, is planned.

Figure 14 shows the simulation of an inclined circle trajectory flown inside a linear vertical wind gradient (shown on the left of the plot), flown inside a thin shear layer (in the middle of the plot), and the 3D trajectories of the two cases with respect to desired reference trajectory to be followed. In presence of a thin wind shear, current control structure fails to correctly handle the wind disturbance for wind velocities we are interested in.

\section{Conclusion}

An experimental demonstration of atmospheric energy-harvesting from horizontal wind gradient in the form of repetitive circles has been made. Leeward side of a ridge has been chosen in order to perform the tests. The feasibility of dynamic soaring in the shear layer with a small autonomous glider has been assessed during these flights. It is experimentally shown that the positive power contribution comes from the segments which are ascending into the positive wind gradient, and descending into the negative wind gradient. Presented flight test results revealed that, without the need of any complex trajectories, on average, the glider can extract close to $60 \%$ of its required power from dynamic soaring. As an additional effort, a 6-DOF simulation environment is presented which can currently simulate various types of atmosphere models and can model the flight of any given aircraft geometry (defined as in AVL input format) for different trajectories such as inclined circular trajectory as performed in flight tests. The measurements that are taken during these flight tests will serve as a database for the researchers to close the gap between simulations and the real flights to achieve a fully autonomous dynamic soaring. The challenges that have to be overcome to achieve autonomous dynamic soaring will certainly improve the overall capabilities of any fixed-wing vehicle (as well as most rotary-wing vehicle).

\section{Appendix}



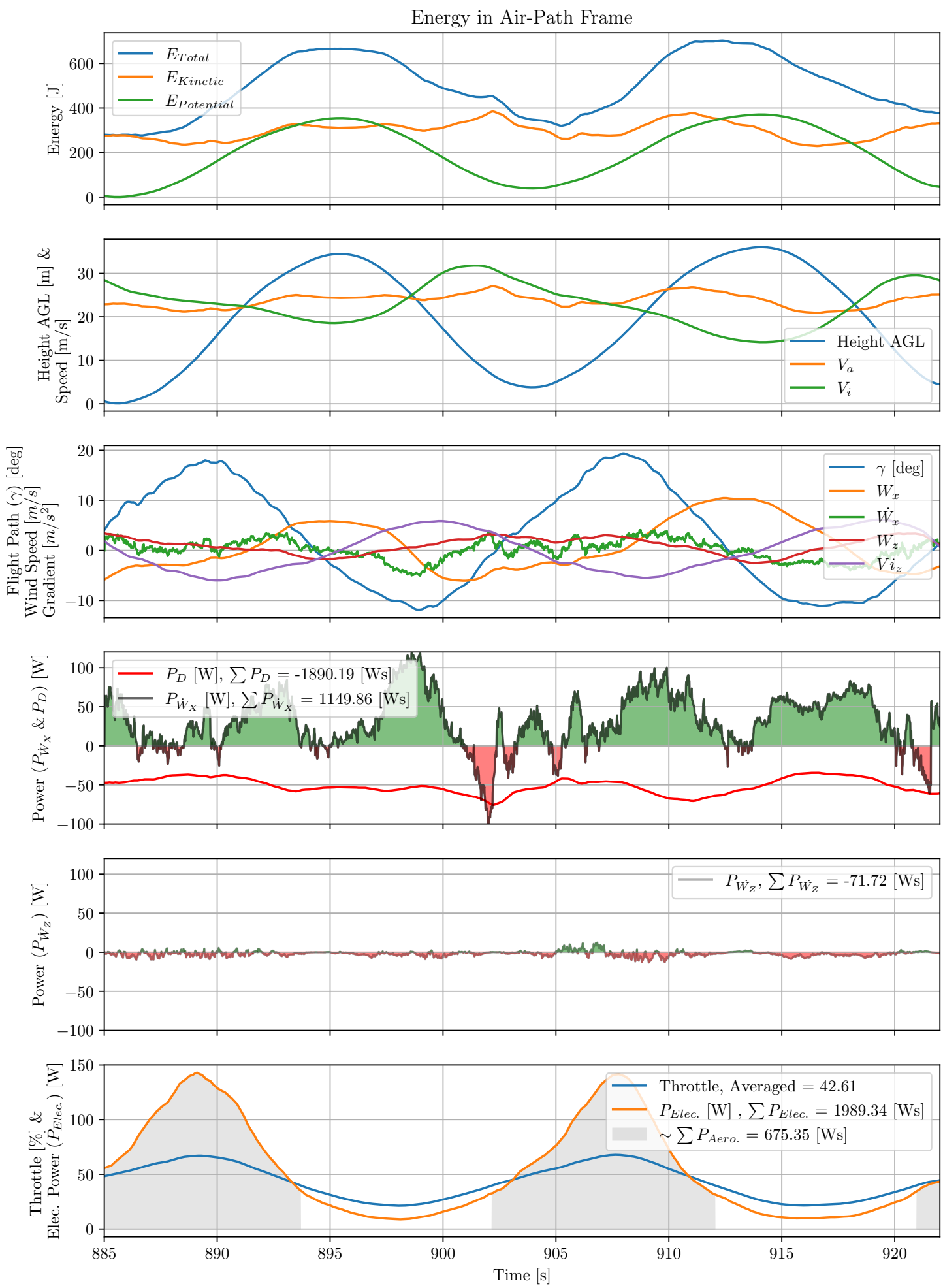

Fig. 15 Two consecutive inclined circles from 20th of November 2020 flight. 

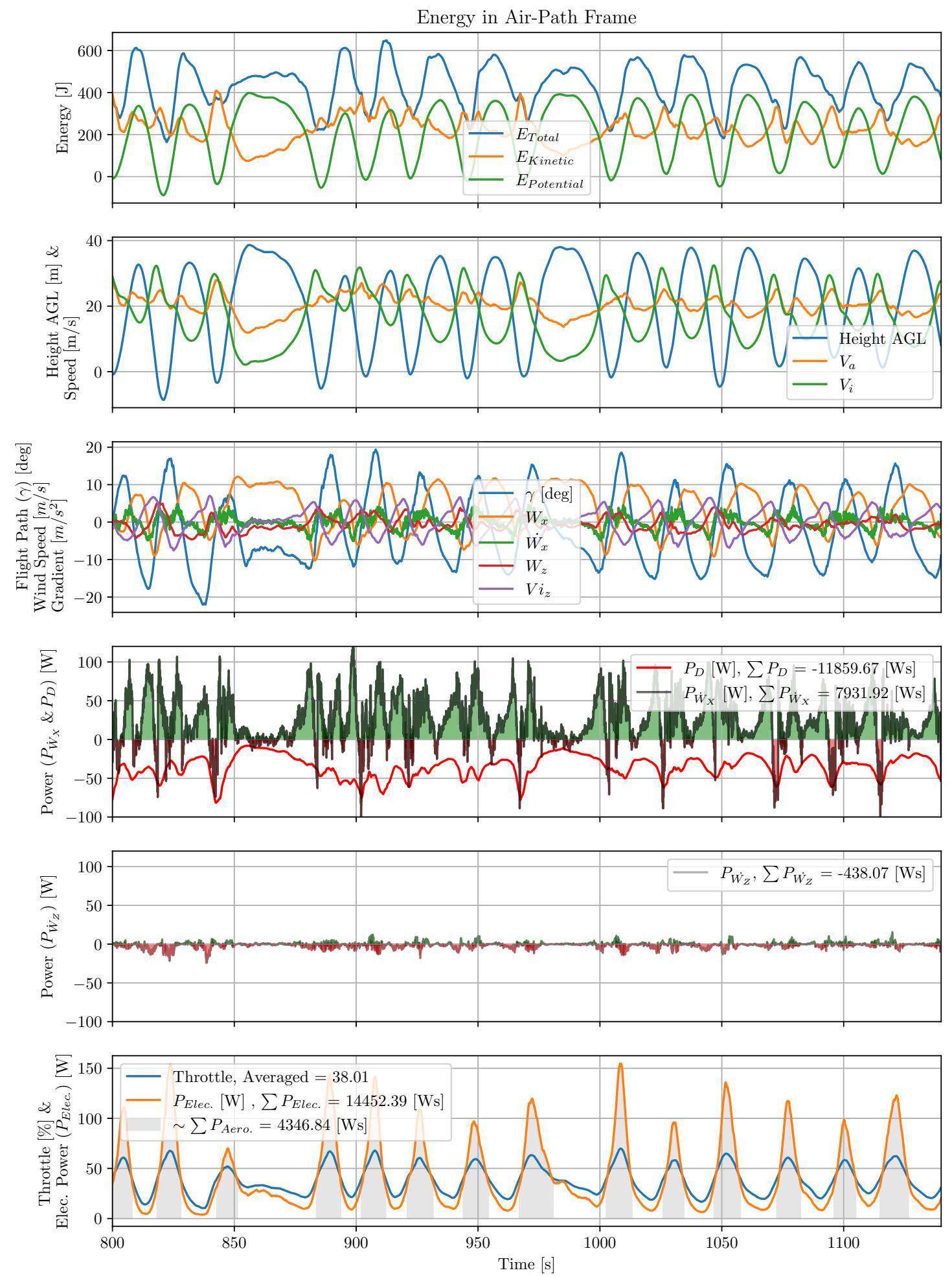

Fig. 16 Fourteen consecutive inclined circle trajectories from 20th of November 2020 flight. 


\section{Acknowledgments}

The authors would like to thank to Michel Gorraz and Alexandre Bustico for the development of Smart-Probe, all related hardware and software, Xavier Paris for his contributions during flight tests and for software modules. Additional thanks goes to the technical team of ISAE-SUPAERO low-Re wind tunnel SABRE, Remy Chanton and Henri Dedieu, for the preparation and realization of Smart-Probe static calibration tests.

\section{References}

[1] Rayleigh, L., “The Soaring of Birds,” Nature, Vol. 28, 1883, pp. 534-535. https://doi.org/10.1038/028198a0

[2] Kai, J.-M., Hamel, T., and Samson, C., "Novel Approach to Dynamic Soaring Modeling and Simulation," Journal of Guidance, Control, and Dynamics, Vol. 42, No. 6, 2019, pp. 1250-1260.

[3] Bousquet, G. D., Triantafyllou, M. S., and Slotine, J.-J. E., "Optimal dynamic soaring consists of successive shallow arcs," Journal of The Royal Society Interface, Vol. 14, No. 135, 2017, p. 20170496.

[4] Sachs, G., and da Costa, O., "Optimization of dynamic soaring at ridges," AIAA Atmospheric flight mechanics conference and exhibit, 2003, p. 5303.

[5] Wurts, J., "Dynamic Soaring,” S\&E Modeller Magazine, Vol. 5, 1998, pp. 2-3.

[6] Fogel, L., "Dynamic Soaring,” S\&E Modeller Magazine, Vol. 5, 1999, pp. 4-7.

[7] Sachs, G. P., "Dynamic Soaring at 600 mph,” AIAA Scitech 2019 Forum, 2019, p. 0107.

[8] Zhao, Y., Dutta, A., Tsiotras, P., and Costello, M., "Optimal Aircraft Trajectories for Wind Energy Extraction,” Journal of Guidance, Control, and Dynamics, Vol. 41, No. 2, 2017, pp. 488-496.

[9] Deittert, M., Richards, A., Toomer, C., and Pipe, A., "Dynamic soaring flight in turbulence," AIAA Guidance, Navigation, and Control Conference, 2009, p. 6012.

[10] Bird, J. J., Langelaan, J. W., Montella, C., Spletzer, J., and Grenestedt, J. L., "Closing the loop in dynamic soaring," AIAA Guidance, Navigation, and Control Conference, 2014, p. 0263.

[11] Boslough, M. B., "Autonomous dynamic soaring platform for distributed mobile sensor arrays," Sandia National Laboratories, Sandia National Laboratories, Tech. Rep. SAND2002-1896, 2002.

[12] Boslough, M., “Autonomous dynamic soaring,” 2017 IEEE Aerospace Conference, IEEE, 2017, pp. 1-20.

[13] Gordon, R. J., "Optimal dynamic soaring for full size sailplanes," Tech. rep., Air Force Inst of Tech Wright-Patterson AFB OH Dept of Aeronautics and ..., 2006.

[14] Gavrilovic, N., Bronz, M., Moschetta, J.-M., and Bénard, E., "Bioinspired wind field estimation-part 1: Angle of attack measurements through surface pressure distribution," International Journal of Micro Air Vehicles, Vol. 10, No. 3, 2018, pp. 273-284.

[15] Gavrilović, N., Bronz, M., and Moschetta, J.-M., "Bioinspired energy harvesting from atmospheric phenomena for small unmanned aerial vehicles," Journal of Guidance, Control, and Dynamics, Vol. 43, No. 4, 2020, pp. 685-699.

[16] Hattenberger, G., Bronz, M., and Gorraz, M., "Using the Paparazzi UAV System for Scientific Research,” 2014 International Micro Air Vehicles Conference and Flight Competition, 2014.

[17] Gavrilovic, N., "Endurance improvement of mini UAVs through energy harvesting from atmospheric gusts," Ph.D. thesis, Toulouse, ISAE-SUPAERO, 2018.

[18] Gavrilovic, N., Mohamed, A., Marino, M., Watkins, S., Moschetta, J.-M., and Benard, E., "Avian-inspired energy-harvesting from atmospheric phenomena for small UAVs," Bioinspiration \& Biomimetics, Vol. 14, No. 1, 2019, pp. 1-20.

[19] Anderson, J. D., Fundamentals of aerodynamics, 5 ${ }^{\text {th }}$ ed., McGraw-Hill, 2011. URL http://www.worldcat.org/isbn/ 9780073398105

[20] Bronz, M., "A contribution to the design of long endurance mini unmanned aerial vehicles," Ph.D. thesis, Toulouse, ISAE-SUPAERO, 2014. 
[21] Bencatel, R., Tasso de Sousa, J., and Girard, A., "Atmospheric flow field models applicable for aircraft endurance extension," Progress in Aerospace Sciences, Vol. 61, 2013, pp. 1 - 25. https://doi.org/https://doi.org/10.1016/j.paerosci.2013.03.001. URL http://www.sciencedirect.com/science/article/pii/S037604211300016X

[22] Langelaan, J., "Long Distance/Duration Trajectory Optimization for Small UAVs," AIAA Guidance, Navigation and Control Conference and Exhibit, 2007. https://doi.org/10.2514/6.2007-6737, URL https://arc.aiaa.org/doi/abs/10.2514/6.2007-6737

[23] Verdu, T., Maury, N., Narvor, P., Seguin, F., Roberts, G., Couvreux, F., Cayez, G., Bronz, M., Hattenberger, G., and Lacroix, S., "Experimental flights of adaptive patterns for cloud exploration with UAVs," International Conference on Intelligent Robots and Systems (IROS 2020), Las Vegas (on line), United States, 2020. URL/https://hal.laas.fr/hal-02994385

[24] Kannan, S., and Johnson, E., "Adaptive Control with a Nested Saturation Reference Model," AIAA Guidance, Navigation, and Control Conference and Exhibit, 2003. https://doi.org/10.2514/6.2003-5324 URL https://arc.aiaa.org/doi/abs/10.2514/6.20035324

[25] De Marina, H. G., Kapitanyuk, Y. A., Bronz, M., Hattenberger, G., and Cao, M., "Guidance algorithm for smooth trajectory tracking of a fixed wing UAV flying in wind flows," 2017 IEEE International Conference on Robotics and Automation (ICRA), IEEE, 2017, pp. 5740-5745. 